\title{
Therapeutic targeting of the MEK/MAPK signal transduction module in acute myeloid leukemia
}

\author{
Michele Milella, ${ }^{1}$ Steven M. Kornblau, ${ }^{1}$ Zeev Estrov, ${ }^{2}$ Bing Z. Carter, ${ }^{1}$ Hélène Lapillonne, ${ }^{1}$ \\ David Harris, ${ }^{2}$ Marina Konopleva, ${ }^{1}$ Shourong Zhao, ${ }^{1}$ Elihu Estey, ${ }^{3}$ and Michael Andreeff ${ }^{1}$
}

${ }^{1}$ Department of Blood and Marrow Transplantation, Section of Molecular Hematology and Therapy,

${ }^{2}$ Department of Bioimmunotherapy, and

${ }^{3}$ Department of Leukemia, The University of Texas, M.D. Anderson Cancer Center, Houston, Texas, USA

Address correspondence to: Michael Andreeff, Section of Molecular Hematology and Therapy,

Department of Blood and Marrow Transplantation, Box 448, The University of Texas,

M.D. Anderson Cancer Center, 1400 Holcombe Boulevard, Houston, Texas 77030, USA.

Phone: (713) 792-7260; Fax: (713) 794-4747; E-mail: mandreef@notes.mdacc.tmc.edu.

Received for publication March 26, 2001, and accepted in revised form August 7, 2001.

The mitogen-activated protein kinase (MAPK) pathway regulates growth and survival of many cell types, and its constitutive activation has been implicated in the pathogenesis of a variety of malignancies. In this study we demonstrate that small-molecule MEK inhibitors (PD98059 and PD184352) profoundly impair cell growth and survival of acute myeloid leukemia (AML) cell lines and primary samples with constitutive MAPK activation. These agents abrogate the clonogenicity of leukemic cells but have minimal effects on normal hematopoietic progenitors. MEK blockade also results in sensitization to spontaneous and drug-induced apoptosis. At a molecular level, these effects correlate with modulation of the expression of cyclin-dependent kinase inhibitors ( $\mathrm{p}^{2} 7^{\mathrm{Kip} 1}$ and $\left.\mathrm{p} 21^{\mathrm{Waf} 1 / \mathrm{CIP} 1}\right)$ and antiapoptotic proteins of the inhibitor of apoptosis proteins (IAP) and Bcl-2 families. Interruption of constitutive MEK/MAPK signaling therefore represents a promising therapeutic strategy in AML.

J. Clin. Invest. 108:851-859 (2001). DOI:10.1172/JCI200112807.

\section{Introduction}

In acute myeloid leukemia (AML), hematopoietic stem cells fail to differentiate normally, resulting in the expansion and accumulation of clonal cells arrested at various stages of development (1). Genetic alterations affecting the function of transcription factors that regulate myeloid maturation play a central role in leukemogenesis (2). In most cases, however, additional genetic alterations are necessary for the leukemic transformation to occur $(3,4)$. In particular, aberrant activation of the kinase-based signal transduction pathways that normally translate extracellular stimuli into appropriate homeostatic responses can powerfully contribute to leukemogenesis by enabling leukemic cells to grow autonomously and escape programmed cell death (5). The mitogen-activated protein kinase (MAPK) pathway is a key integration point along the signal transduction cascade that links diverse extracellular stimuli to proliferation, differentiation, and survival (6). Constitutive activation of the MAPK pathway drives the oncogenic transformation of normal fibroblasts and is commonly detected in human cancers resulting from a variety of genetic alterations, from ras mutations to the overexpression of growth factor receptors $(6,7)$. Inappropriate MAPK activation may also play a role in the leukemic transformation of myeloid cells. Activation of upstream kinases (MAPK kinase, also known as MEK), downregulation of phosphatases, and overexpression of $\mathrm{p} 44 / 42^{\mathrm{MAPK}}$ (also known as extracellular signal-regulated kinase $1 / 2$, hereafter referred to as MAPK) itself result in constitutive MAPK activation in most AML cases $(8,9)$. Internal tandem duplications of the Flt3 receptor also result in constitutive MAPK activation and the autonomous growth of myeloid cell lines and primary AML samples (10). Direct evidence for a role of the MAPK pathway in leukemic transformation comes from the findings that a conditionally active form of MEK1 converts hematopoietic cells to cytokine independence $(11,12)$ and that the efficient induction of a myeloproliferative phenotype by TEL-TRKC fusion variants depends critically on their ability to activate the MAPK pathway (13).

Recent data from our group indicate that constitutive MAPK phosphorylation is an independent predictor of poor response to chemotherapy and shorter survival in AML patients (S.M. Kornblau, unpublished observations). Here we demonstrate that disruption of the MEK/MAPK module has profound functional consequences in AML cell lines and primary AML samples, while it is relatively sparing of normal hematopoietic progenitors, thus providing the preclinical rationale for the development of MAPK-targeted therapeutic strategies in AML.

\section{Methods}

Cell lines, primary samples, and cell cultures. AML cell lines were cultured under standard conditions (14). Bone marrow and peripheral blood samples were obtained from AML patients and healthy donors after informed consent, according to institutional guidelines. Mononuclear 
cells were purified by Ficoll-Hypaque (Sigma Chemical Co., St. Louis, Missouri, USA) density-gradient centrifugation. $\mathrm{CD} 34^{+}$cells were separated to greater than $95 \%$ purity by positive-selection magnetic-bead sorting using a VarioMACS device (Miltenyi Biotec, Auburn, California, USA). Cell lines were harvested in log-phase growth and exposed to the MEK inhibitors PD98059 (2'-amino-3'methoxyflavone; Calbiochem-Novabiochem Corp., La Jolla, California, USA) $(15,16)$ and PD184352 (2-[2chloro-4-iodo-phenylamino]- $N$-cyclopropylmethoxy-3,4difluoro-benzamide) (17), or to a matched concentration of vehicle (DMSO). In combination experiments, cells were preincubated with PD98059 for 1 hour prior to the addition of all-trans-retinoic acid (ATRA). In other experiments, cells were seeded at $5 \times 10^{5}$ cells $/ \mathrm{ml}$ and exposed to $1-\beta$-D-arabinofuranosylcytosine (Ara-C) for 4 hours at $37^{\circ} \mathrm{C}$. Ara-C was then removed and cells were plated at 1 $\times 10^{5}$ cells $/ \mathrm{ml}$ in the presence of either DMSO or PD98059. In all experiments, cell viability was evaluated by triplicate counting of trypan blue dye-excluding cells under a light microscope. AML blast and normal bone marrow progenitor colony assays were performed as previously described (18).

Cell cycle and apoptosis analysis. Cells were fixed in icecold ethanol $(70 \% \mathrm{vol} / \mathrm{vol})$ and stained with propidium iodide (PI) solution $(25 \mu \mathrm{g} / \mathrm{ml}$ PI, $180 \mathrm{U} / \mathrm{ml}$ RNase, $0.1 \%$ Triton $\mathrm{X}-100$, and $30 \mathrm{mg} / \mathrm{ml}$ polyethylene glycol in $4 \mathrm{mM}$ citrate buffer, $\mathrm{pH}$ 7.8; Sigma Chemical Co.). The DNA content was determined using a FACScan flow cytometer (Becton Dickinson Immunocytometry Systems, San Jose, California, USA). Cell cycle distribution was analyzed using ModFit LT software (Verity Software House Inc., Topsham, Maine, USA). Cells with a hypodiploid DNA content $(<2 n,>0.2 n)$ were counted as apoptotic. For annexin $V$ binding studies, cells were washed twice with binding buffer $(10 \mathrm{mM}$ HEPES, $140 \mathrm{mM} \mathrm{NaCl}$, and $5 \mathrm{mM} \mathrm{CaCl}_{2}$ at $\mathrm{pH}$ 7.4; Sigma Chemical Co.) and incubated with a 1:500 solution of FITC-conjugated annexin V (Roche Diagnostic Corp., Indianapolis, Indiana, USA) for 15 minutes at room temperature. Stained cells were analyzed by flow cytometry, while membrane integrity was simultaneously assessed by PI exclusion.

Western blot analysis and in vitro kinase assay. For phosphorylation studies, cells were lysed for 10 minutes on ice in a solution containing $10 \mathrm{mM} \mathrm{NaF}, 1 \mathrm{mM}$ $\mathrm{Na}_{3} \mathrm{VO}_{4}, 150 \mathrm{mM} \mathrm{NaCl}, 1 \mathrm{mM} \mathrm{MgCl}, 1 \mathrm{mM} \mathrm{CaCl}$, $0.1 \% \mathrm{NaN}_{3}, 10 \mathrm{mM}$ iodoacetamide, $3 \mathrm{mM} \mathrm{PMSF}$, and $1 \%$ Triton X-100, supplemented with a protease inhibitor cocktail (Roche Diagnostic Corp.). Equal amounts of proteins were subjected to SDS-PAGE, transferred to Hybond-P membranes (Amersham Pharmacia Biotech, Little Chalfont, England), and immunoblotted with an $\mathrm{mAb}$ specific for T202/Y204 doubly-phosphorylated p44/42 MAPK (Cell Signaling Technology Inc., Beverly, Massachusetts, USA). Blots were then stripped and reprobed with a polyclonal $\mathrm{Ab}$ recognizing p42 ${ }^{\mathrm{MAPK}}$ (Santa Cruz Biotechnology Inc., Santa Cruz, California, USA). Survivin immunoblot- ting was performed as previously described (14). For proteins related to cell cycle and apoptosis, the following primary Ab's were used: anti-phospho-p27 $7^{\text {Kip } 1 ~}$ (T187) rabbit polyclonal Ab (Zymed Laboratories Inc.,

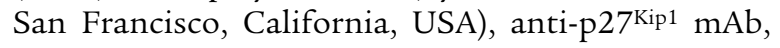
anti-Mcl-1 mAb (both from PharMingen, San Diego, California, USA), anti-Waf-1 mAb, anti-Bcl- $\mathrm{X}_{\mathrm{L}} \mathrm{mAb}$ (both from Oncogene Research Products, Cambridge, Massachusetts, USA), and anti-Bcl-2 mAb (DAKO Corp., Carpinteria, California, USA). MAPK enzymatic activity was assessed by immunoprecipitation and in vitro kinase assay using the p44/42 MAP Kinase Assay Kit (Cell Signaling Technology Inc.).

Quantitative real-time RT-PCR. OCI-AML3 cells were treated with either DMSO or PD98059, and RNA was isolated with TRIzol solution (Life Technologies Inc., Grand Island, New York, USA). One microgram of total RNA was reverse transcribed by AMV reverse transcriptase (Roche Diagnostic Corp.) at $42^{\circ} \mathrm{C}$ for 1 hour. PCR amplification reaction mixtures $(25 \mu \mathrm{l})$ contained cDNA, p21 Waf1/CIP1 (5'-CGCTAATGGCGGGCTG-3') or p27 Kip1 (5'-GAGGACACGCATTTGGTGG-3') forward primers, p21 Waf1/CIP1 (5'-CGGTGACAAAGTCGAAGTTCC$\left.3^{\prime}\right)$ or p27 $7^{\text {Kip } 1}$ (5'-GCTCCGCTAACCCCGTCT-3') reverse primers, p21 Waf1/CIP1 (5'-ATCCAGGAGGCCCGTGAGCGA$\left.3^{\prime}\right)$ or p27 $7^{\text {Kip } 1}$ (5'-CCCAAAGACTGATCCGTCGGACAGC-3') probes, and TaqMan Universal PCR Master Mix (PE Applied Biosystems, Foster City, California, USA). $\beta_{2^{-}}$ microglobulin $\left(\beta_{2}-\mathrm{m}\right)$ coamplified with $\mathrm{p} 21^{\mathrm{Waf} 1 / \mathrm{CIP} 1}$ and p27 Kip1 was included as internal control for normalization of the variable content of cDNA in each sample (forward primer 5'-AGCTGTGCTCGCGCTACTCT- $3^{\prime}$, reverse primer $5^{\prime}$-TTGACTTTCCATTCTCTGCTGG-3', probe $5^{\prime}$-TCTTTCTGGCCTGGAGGGCATCC- ${ }^{\prime}$ ). Thermal cycle conditions included holding the reactions at $50^{\circ} \mathrm{C}$ for 2 minutes and at $95^{\circ} \mathrm{C}$ for 10 minutes, and cycling for 40 cycles between $95^{\circ} \mathrm{C}$ for 15 seconds and $60^{\circ} \mathrm{C}$ for 1 minute. Results were collected and analyzed with an ABI Prism 7700 Sequence Detection System (PE Applied Biosystems) as follows: the PCR cycle number that generated the first fluorescence signal above a threshold (threshold cycle, $\mathrm{C}_{\mathrm{T}} ; 10$ SDs above the mean fluorescence generated during the baseline cycles) was determined, and a comparative $\mathrm{C}_{\mathrm{T}}$ method was then used to measure relative gene expression. The following formula was used to calculate the relative amount of the transcript of interest in the treated sample (X) and the control sample $(\mathrm{Y})$, both normalized to an endogenous reference $\left(\beta_{2}-\mathrm{m}\right): 2^{-\Delta \Delta \mathrm{CT}}$, where $\Delta \mathrm{C}_{\mathrm{T}}$ is the difference in $C_{T}$ between the gene of interest and $\beta_{2}-\mathrm{m}$, and $\Delta \Delta \mathrm{C}_{\mathrm{T}}$ for sample $\mathrm{X}=\Delta \mathrm{C}_{\mathrm{T}}(\mathrm{X})-\Delta \mathrm{C}_{\mathrm{T}}(\mathrm{Y})$.

\section{Results}

MEK blockade inbibits the growth of AML cell lines and primary AML samples. We analyzed MAPK expression and phosphorylation status in freshly isolated, unstimulated blasts from 186 patients with newly diagnosed AML. Constitutive MAPK phosphorylation was detectable in 138 samples $(74 \%)$, indicating that this pathway is dereg- 
a

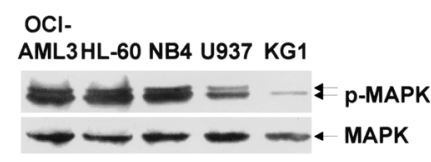

b

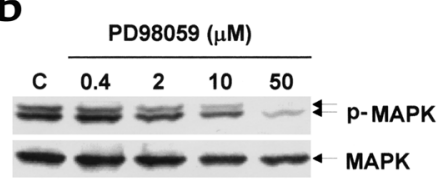

C

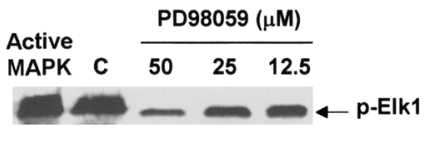

d

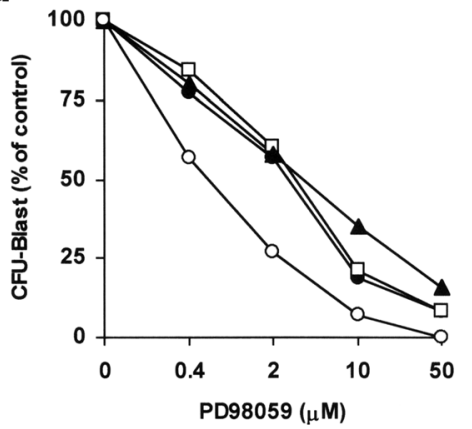

e

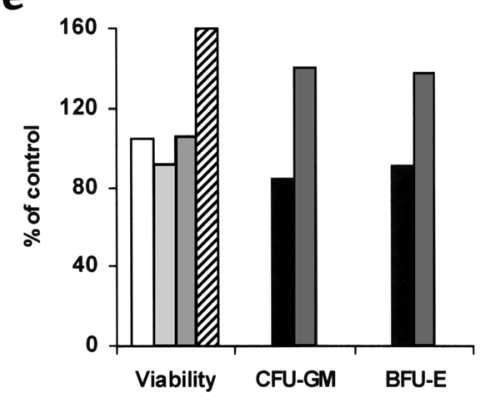

\section{Figure 1}

MEK blockade inhibits the growth of leukemic, but not normal, hematopoietic cells. (a) AML cell lines were assessed for MAPK phosphorylation by Western blot. (b) OCI-AML3 cells were exposed to DMSO (C) or PD98059 at the indicated doses for 6 hours. MAPK phosphorylation was then assessed by Western blot. (c) OCI-AML3 cells were treated as in b. MAPK enzymatic activity was measured by the ability to phosphorylate the specific substrate Elk1 in an in vitro kinase assay. (d) Mononuclear cells from patients 5 (filled circles), 9 (open squares), 10 (filled triangles), and 11 (open circles) were grown in colony assays in the presence of DMSO or PD98059 at the indicated doses. Results show AML blast colonies in the PD98059-treated group, expressed as a percentage of the colonies in the DMSO-treated group, and represent the average of quadruplicate cultures (SEM was constantly less than 10\%). (e) MACS-sorted CD34+ cells from healthy donors were cultured in the presence of DMSO or 20 $\mu \mathrm{M}$ PD98059. Viability was then assessed by trypan blue dye exclusion. Results show viable cells in the PD98059-treated group, expressed as a percentage of the viable cells in the DMSO-treated group (four bars at left; each bar represents a single donor). Bone marrow cells from healthy donors were grown in colony assays in the presence of DMSO or $50 \mu \mathrm{M}$ PD98059. Results show CFU-GM and burst-forming units-erythroid (BRU-E) in the PD98059-treated group, expressed as a percentage of the colonies in the DMSO-treated group (four bars at right; two donors).

ulated in most AML patients (S.M. Kornblau, unpublished observations). We therefore investigated the functional consequences of the pharmacological disruption of the MEK/MAPK module using the selective inhibitor of MEK activation PD98059. Among the AML cell lines tested, OCI-AML3, HL-60, and NB4 showed high steadystate levels of doubly phosphorylated (i.e., active) MAPK (Figure 1a). MAPK activation appeared to be constitutive in that comparably high levels of MAPK phosphorylation were detectable even after 48 hours of serum starvation (data not shown). As shown in Figure 1, b and c, PD98059 inhibited MAPK phosphorylation and enzymatic activity downstream of MEK in OCI-AML3 cells in a dose-dependent manner. MEK blockade resulted in a profound, dose-dependent growth inhibition in OCIAML3 and HL-60 cells, and a less extensive inhibition in NB4 cells (Table 1). Conversely, U937 and KG1 cells, which showed low levels of constitutive MAPK activation (Figure 1a), proved relatively resistant (Table 1).

In primary AML samples, PD98059 $(20 \mu \mathrm{M})$ caused a greater than $25 \%$ decrease in the recovery of viable cells after short-term in vitro culture in 14 of 19 samples tested (median reduction in sensitive samples, $40 \%$; range, 26-60\%) (Table 2). MEK blockade by PD98059 profoundly affected primary AML cell growth and viability at the progenitor cell level, as shown by the striking dosedependent decrease in the number of AML blast colonies observed in all four samples tested (Figure 1d). Con- versely, no reduction in either cell viability or clonogenic potential was observed in response to MEK blockade in CD34-selected progenitors $(n=4)$ and bone marrow cells $(n=2)$ from healthy donors (Figure 1e).

Collectively, these findings indicate that disruption of the MEK/MAPK module selectively inhibits the growth of AML cell lines and primary samples, but not that of normal hematopoietic progenitors.

MEK blockade inhibits cell cycle progression. Consistent with the observed inhibition of cell growth, PD98059 caused a dose- and time-dependent inhibition of $\mathrm{G}_{1} / \mathrm{S}$ transition, with accumulation of cells in the $G_{1}$ phase in MEK inhibition-sensitive cell lines and primary samples (Figure 2, a and d). Conversely, the resistant cell lines U937 and KG1 showed a slight inhibition of cell cycle progression only at the highest dose (data not shown). Western blot analysis showed that MEK inhibition-induced cell cycle arrest was accompanied by downregulation of $\mathrm{p} 21^{\text {Waf } 1 / \mathrm{CIP} 1}$ and upregulation of $\mathrm{p} 27^{\mathrm{Kip} 1}$ in sensitive cell lines (OCI-AML3 and HL-60), but not in resistant cell lines (U937) (Figure 2b). Levels of $\mathrm{p} 21^{\text {Waf1/CIP1 }} \mathrm{RNA}$ decreased strikingly in response to PD98059 in a timedependent fashion (Figure 2c), suggesting the possibility of direct transcriptional regulation (19). Conversely, p27 ${ }^{\text {Kip } 1}$ RNA levels remained relatively constant following exposure to PD98059. However, T187 phosphorylation, a posttranslational modification that targets p27Kip1 for proteasome-mediated degradation (20), was already 
Table 1

$\mathrm{IC}_{50}$ of MEK inhibitors in AML cell lines

\begin{tabular}{lcc} 
Cell line & A & \multicolumn{2}{c}{$\mathrm{IC}_{50}(\mu \mathrm{M})$} \\
& PD98059 & PD184352 \\
OCl-AML3 & 5.4 & 0.06 \\
HL-60 & 5.5 & 0.07 \\
NB4 & 16.7 & 0.8 \\
U937 & 59.2 & 5.5 \\
KG1 & 33.2 & 6.7
\end{tabular}

${ }^{A} A M L$ cell lines were cultured under standard conditions in the presence of DMSO or escalating doses of either PD98059 or PD184352. After 96 hours, live cells were counted by trypan blue dye-exclusion and $\mathrm{IC}_{50}$ was calculated for each treatment.

decreased by PD98059 after 2 hours, suggesting that protein stabilization occurs before changes in cell cycle distribution are detectable (Figure $2 \mathrm{~d}$ ).

$M E K$ inbibition induces apoptosis in AML cell lines and primary $A M L$ samples. Consistent with the reported reversibility of MEK inhibition, removal of PD98059 from the culture medium promptly restored cell cycle progression and growth in HL-60 and NB4 cells (data not shown). By contrast, OCI-AML3 cells showed sustained growth impairment even 96 hours after PD98059 removal (data not shown). Since the MEK/MAPK pathway is considered a key regulator of apoptotic cell death $(21,22)$, we tested the hypothesis that sustained growth impairment might be due to induction of apoptosis. Treatment of OCI-AML3 cells with $20 \mu \mathrm{M}$ PD98059 caused a time-dependent increase in the percentage of annexin $\mathrm{V}$-binding cells that was detectable within 48 hours, and reached maximum levels after 96 hours (Figure 3a). Phosphatidylserine exposure on the outer leaflet of the plasma membrane was accompanied by caspase- 3 activation and a decrease in the DNA content to hypodiploid levels (data not shown). A slight increase in apoptosis was also induced by PD98059 in HL-60 cells, but not in the other cell lines tested (data not shown). A net increase in the proportion of apoptotic cells of more than $10 \%$, as detected by annexin $\mathrm{V}$ binding and hypodiploid DNA content, was observed in 11 of 18 primary AML samples after exposure to $20 \mu \mathrm{M}$ PD98059 (median net apoptosis increase in sensitive samples, 13\%; range, $10-23 \%$; data not shown). The dose dependency of PD98059-induced apoptosis in MEK inhibition-sen- a
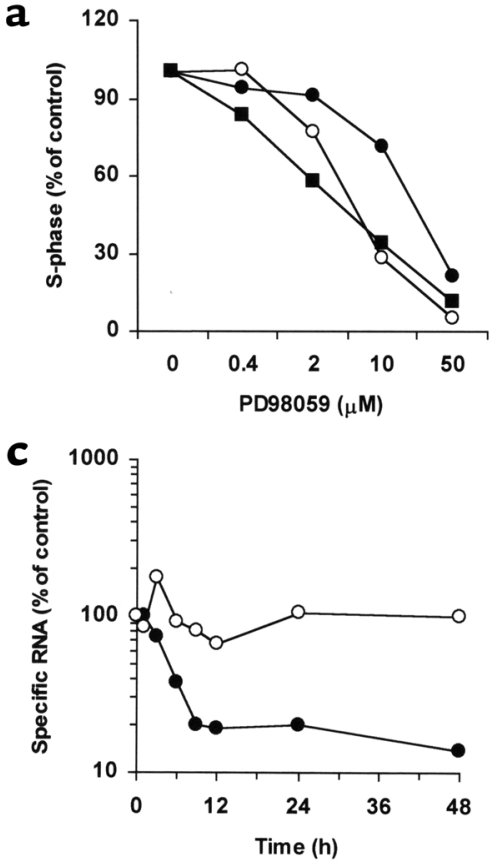
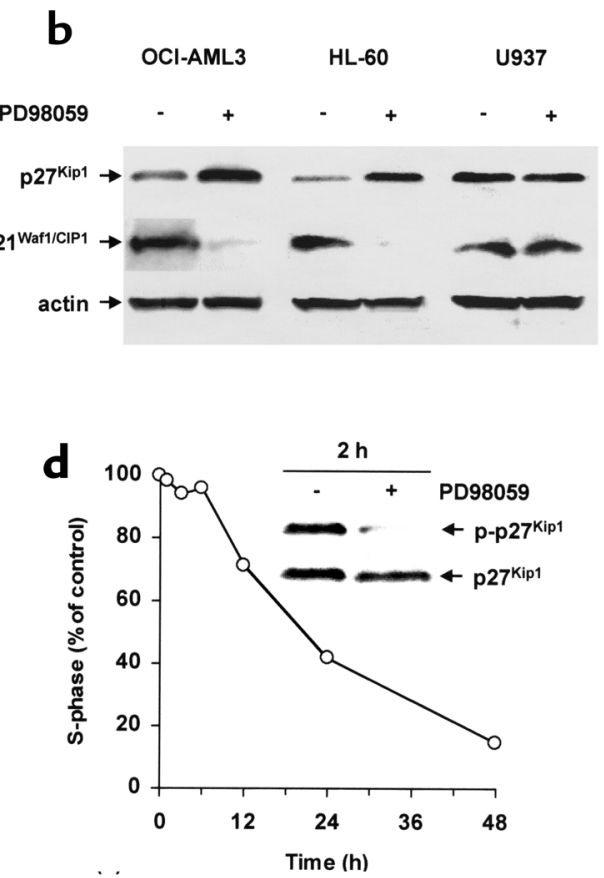

Figure 2

MEK inhibition causes cell cycle arrest and modulates $\mathrm{p} 27^{\mathrm{Kip} 1}$ and $\mathrm{p} 21^{\text {Waf1/CIP1 }}$ expression. (a) OCI-AML3 (open circles), HL-60 (filled circles), and freshly isolated leukemic blasts from patient 3 (filled squares) were cultured for 48 hours in the presence of DMSO or PD98059 at the indicated concentrations, and then stained for DNA content. Results are expressed as percentage of S-phase cells in the DMSO-treated group. Cell line results are representative of one of three independent experiments. (b) OCI-AML3, HL-60, and U937 cells were cultured for 24 hours in the presence of DMSO or PD98059 $(20 \mu \mathrm{M})$, and subjected to Western blot analysis with mAb's specific for the indicated cell cycle-regulating proteins. Results are representative of one of three independent experiments. (c) OCI-AML3 cells were cultured for the indicated times in the presence of DMSO or PD98059 $(20 \mu \mathrm{M})$, and subjected to quantitative real-time PCR analysis of p21 Waf1/CIP1 (filled circles) and p27 Kip1 (open circles) RNA transcripts. Results are expressed as percentage of specific RNA transcripts in the DMSO-treated group and are representative of one of two independent experiments. (d) OCl-AML3 cells were cultured for the indicated times in the presence of DMSO or PD98059 $(20 \mu \mathrm{M})$ and assessed for DNA content. Results are expressed as percentage of S-phase cells in the DMSO-treated group. Two hours after the addition of PD98059, cells were lysed, and the phosphorylation status of p27 Kip1 was analyzed by Western blot using T187-phosphospecific Ab's (p-p27Kip1). 
a

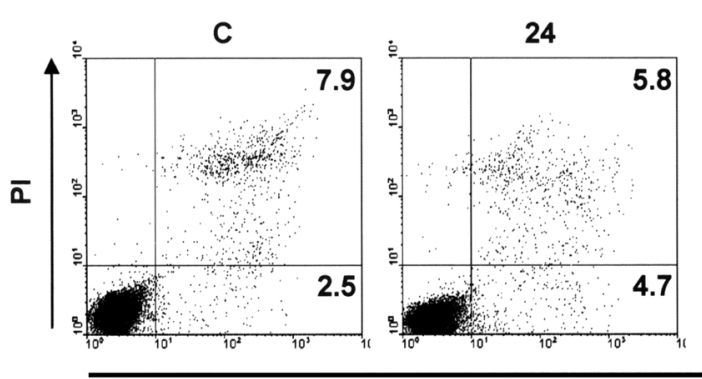

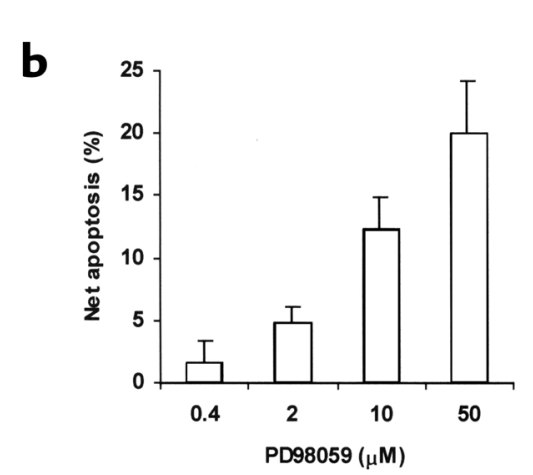
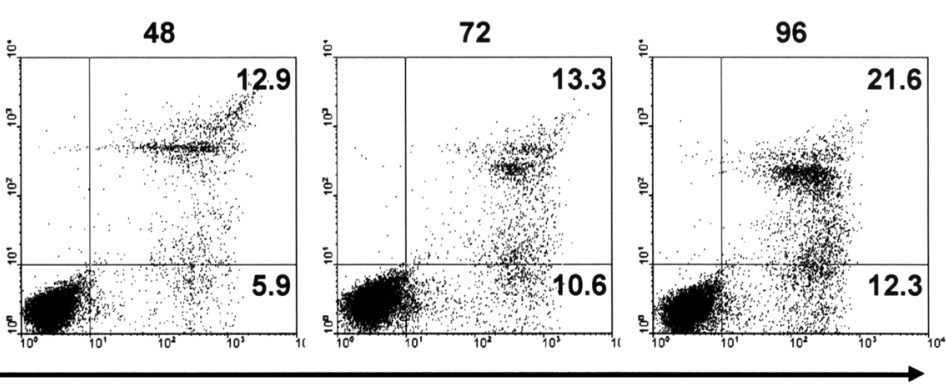

Annexin V
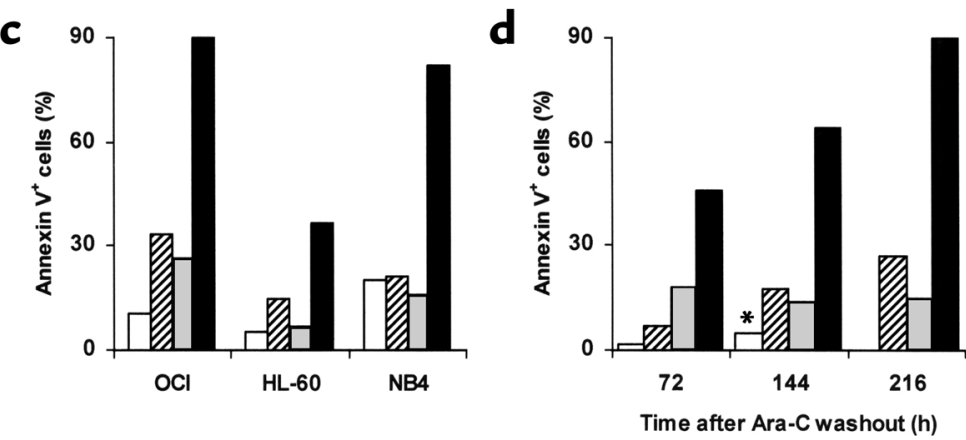

Figure 3

MEK inhibition sensitizes to spontaneous and drug-induced apoptosis. (a) OCI-AML3 cells were cultured in the presence of DMSO or PD98059 $(20 \mu \mathrm{M})$ and stained for annexin V binding at the indicated times. Matched DMSO control at 96 hours is shown (C). Results are representative of one of three independent experiments. (b) Primary AML blasts were cultured in the presence of DMSO or PD98059 at the indicated doses for 96 hours. Apoptosis was then measured as percentage of cells with hypodiploid DNA content. Results are expressed as the net apoptosis induction (percentage of apoptosis in treated cells minus percentage of apoptosis in DMSO-treated cells) and represent the mean \pm SD of the results obtained in three different patient samples (patients 3,14, and 16). (c) AML cell lines were cultured for 96 hours in the presence of DMSO (white bars), $20 \mu \mathrm{M}$ PD98059 (hatched bars), $1 \mu \mathrm{M}$ ATRA (gray bars), or a combination of PD98059 and ATRA (black bars), and stained for annexin $\mathrm{V}$ binding. Results are representative of one of three independent experiments. (d) HL-60 cells were exposed to $10 \mu \mathrm{M}$ Ara-C, washed, and cultured in the presence of DMSO (gray bars) or $20 \mu \mathrm{M}$ PD98059 (black bars). White bars and hatched bars represent DMSO- and PD98059-treated HL-60 cells, respectively, in the absence of prior exposure to Ara-C. At the indicated times, cells were stained for annexin $\mathrm{V}$ binding. ${ }^{*} \mathrm{DMSO}$-treated cultures were discontinued after 144 hours due to overgrowth. Results are representative of one of three independent experiments.

sitive samples $(n=3)$ is shown in Figure 3b. Conversely, no induction of apoptosis, but rather a slight protection from spontaneous cell death in cytokine-free cultures, was observed in normal $\mathrm{CD} 34^{+}$progenitor cells from healthy donors following treatment with PD98059 (20 $\mu \mathrm{M} ; n=4)$ (data not shown).

MEK inhibition sensitizes AML cells to retinoid- and chemotherapy-induced apoptosis. PD98059 $(20 \mu \mathrm{M})$ also potently sensitized AML cell lines with constitutive MAPK activation (OCI-AML3, NB4, HL-60), but not those with low steady-state levels of MAPK activity (U937, KG1), to ATRA-induced apoptosis (Figure 3c). Apoptosis was not caused by increased differentiation; on the contrary, ATRA-induced differentiation was partially blocked by simultaneous MEK inhibition, as previously reported (23). A similar degree of sensitization was observed in response to the pan-RA receptor agonist 9-cis-RA, but not to RAR- or RXR-selective ligands, suggesting that this proapoptotic effect requires simultaneous activation of both the RAR and RXR pathways (data not shown). Moreover, when HL-60 cells were exposed to clinically relevant concentrations $(10 \mu \mathrm{M})$ of Ara-C for short periods (4 hours), the subsequent exposure to
PD98059 $(20 \mu \mathrm{M})$ greatly potentiated drug-induced apoptosis (Figure $3 \mathrm{~d}$ ), and completely prevented regrowth of the leukemic cells for up to 9 days. This effect was strictly sequence dependent, in that pretreatment with PD98059 did not sensitize leukemic cells to, but rather slightly protected them from Ara-C-induced killing (data not shown). Similar results were obtained in OCI-AML3 and NB4 cells, whereas no such effect was observed in U937 and KG1 cells (data not shown).

MEK inhibition downregulates antiapoptotic proteins of the "inhibitor of apoptosis protein" and Bcl-2 families. In an attempt to elucidate the molecular mechanisms underlying the lowering of the apoptotic threshold in response to MEK blockade, we examined whether PD98059 affected the expression of antiapoptotic proteins of the "inhibitor of apoptosis protein" (IAP) and Bcl-2 families. We have recently reported that survivin expression is regulated through MAPK-dependent mechanisms in OCI-AML3 cells (14). Here we show that survivin levels were significantly decreased by treatment with $20 \mu \mathrm{M}$ PD98059 in the MEK inhibition-sensitive cell lines (OCI-AML3 and HL-60), but not in the resistant cell lines (U937; Figure 4a). Overall, a strong, statis- 
Table 2

Effect of MEK inhibition in freshly isolated AML blasts

\begin{tabular}{|c|c|c|c|c|c|c|}
\hline Patient no. & Sex & FAB & Cytogenetics & Sample & Blasts (\%) & $\begin{array}{c}\text { Cell number } \\
\text { reduction }(\%)^{\mathrm{A}}\end{array}$ \\
\hline 1 & $\mathrm{~F}$ & M4 & Diploid & PB & 62 & 60 \\
\hline 2 & M & M1 & Diploid & PB & 64 & 30 \\
\hline 3 & $M$ & M2 & -7 & PB & 48 & 51 \\
\hline 4 & $M$ & M4 & Diploid & BM & 81 & 59 \\
\hline 5 & M & Previous MDS & $+8,-12 ;+8$ & PB & 95 & 24 \\
\hline 6 & M & M2 & $\mathrm{t}(8 ; 21)(\mathrm{q} 22 ; \mathrm{q} 22)$ & PB & 80 & 26 \\
\hline 7 & $\mathrm{~F}$ & $\mathrm{~N} / \mathrm{A}$ & Diploid & PB & 41 & 20 \\
\hline 8 & M & M0 & $\mathrm{t}(2 ; 11)(\mathrm{p} 21 ; \mathrm{q} 25)$ & BM & 77 & 32 \\
\hline 9 & $\mathrm{~F}$ & M2 & $\mathrm{t}(8 ; 21)(\mathrm{q} 22 ; \mathrm{q} 22)$ & PB & 70 & $\mathrm{~N} / \mathrm{A}$ \\
\hline 10 & M & M2 & $\mathrm{t}(7 ; 11)(\mathrm{p} 15 ; \mathrm{p} 15), \mathrm{i}(17)(\mathrm{q} 10)$ & BM & 95 & 28 \\
\hline 11 & $\mathrm{~F}$ & M2 & Diploid & BM & 76 & $\mathrm{~N} / \mathrm{A}$ \\
\hline 12 & M & $\mathrm{N} / \mathrm{A}$ & $+13 ;-5, \operatorname{add}(6)(\mathrm{p} 24),+13$ & BM & 50 & 40 \\
\hline 13 & $\mathrm{~F}$ & $\mathrm{~N} / \mathrm{A}$ & No mitotic cells recovered & PB & 62 & 34 \\
\hline 14 & $\mathrm{~F}$ & M2 & Diploid & PB & 89 & 48 \\
\hline 15 & $\mathrm{~F}$ & M5A & del(11)(q23); Tetraploid clone & PB & 65 & 19 \\
\hline \multirow[t]{2}{*}{16} & M & M3 & $\operatorname{inv}(9)(p 11 q 12), t(15 ; 17)(q 22 ; q 21)$ & PB & 78 & 41 \\
\hline & & & & BM & 78 & 39 \\
\hline \multirow[t]{2}{*}{17} & M & M5A & $+11 ;+11,+13$ & PB & 98 & 13 \\
\hline & & & & BM & 88 & 36 \\
\hline 18 & M & M1 & $\operatorname{del}(5)(q 22 q 35)$ & BM & 94 & 14 \\
\hline \multirow[t]{2}{*}{19} & M & M4 & $+8 ;+8,+19 ;+3,+8,+19$ & BM & 79 & 51 \\
\hline & & & & & & $49^{B}$ \\
\hline 20 & $\mathrm{~F}$ & $\mathrm{~N} / \mathrm{A}$ & Diploid & PB & 52 & $10^{\mathrm{B}}$ \\
\hline 21 & $\mathrm{~F}$ & M2 & Diploid & PB & 80 & $36^{\mathrm{B}}$ \\
\hline 22 & M & M0 & $\mathrm{t}(2 ; 11)(\mathrm{p} 21 ; \mathrm{q} 25)$ & PB & 83 & $23^{B}$ \\
\hline 23 & $\mathrm{~F}$ & M1 & $\operatorname{ins}(3 ; 3)(q 26 ; q 21 q 26),-7$ & PB & 77 & $54^{\mathrm{B}}$ \\
\hline 24 & M & $\mathrm{N} / \mathrm{A}$ & Diploid & PB & 92 & $56^{\mathrm{B}}$ \\
\hline 25 & M & Previous MDS & -7 & BM & 51 & $41^{\mathrm{B}}$ \\
\hline 26 & $\mathrm{~F}$ & M4 & Diploid & BM & 61 & $54^{\mathrm{B}}$ \\
\hline 27 & M & M5 & +8 & BM & 84 & $57^{\mathrm{B}}$ \\
\hline
\end{tabular}

ALive cells were counted by trypan blue exclusion after 48-96 hours of treatment with either DMSO or PD98059 (20 $\mu$ M). The percent reduction in the recovery of live cells was calculated as follows: cell number reduction $(\%)=[1$ - cell number $(\mathrm{PD} 98059) /$ cell number $(\mathrm{DMSO})] \times 100 .{ }^{\mathrm{B} P D 184352}(10 \mu \mathrm{M})$. FAB, French-American-British classification; PB, peripheral blood; BM, bone marrow; MDS, myelodysplastic syndrome.

tically significant correlation was observed between constitutive MAPK activation and the reduction in survivin levels in response to MEK inhibition $\left(R^{2}=0.9104\right.$, $P=0.012$; Figure $4 \mathrm{~b}$ ). Antiapoptotic protein XIAP levels were only slightly affected. PD98059 also significantly decreased Mcl-1 expression, whereas no changes in $\mathrm{Bcl}-2$ and $\mathrm{Bcl}-\mathrm{X}_{\mathrm{L}}$ levels were detected, with the notable exception of HL-60 cells, in which Bcl- $\mathrm{X}_{\mathrm{L}}$ became undetectable within 48 hours (Figure $4 a$ ).

Effects of the novel MEK inhibitor PD184352 in AML cell lines and primary samples. Sebolt-Leopold et al. have recently reported that the novel, orally bioavailable MEK inhibitor PD184352 successfully suppresses the growth of colon tumors in vivo without causing overt toxicity (17). We therefore tested its effects in AML cell lines and primary AML samples. Consistent with results reported in different cellular models $(17,24)$, PD184352 inhibited MAPK phosphorylation and cell growth at nanomolar concentrations in sensitive cell lines (Figure 5, a and b; Table 1). In primary AML samples, PD184352 $(10 \mu \mathrm{M})$ caused a greater than $25 \%$ decrease in the recovery of viable cells in seven of nine samples tested (median, 54\%; range, 36$-57 \%$; Table 2). The pattern of sensitivity to the growthinhibiting effects of PD184352 was superimposable on that observed with PD98059 in primary samples tested for response to both MEK inhibitors $(n=5$; Table 2 and data not shown). PD184352 caused $\mathrm{G}_{1}$ arrest (Figure $5 \mathrm{c}$ ), and at higher doses $(1-10 \mu \mathrm{M})$, induced apoptotic cell death in cells with constitutive MAPK activation (OCIAML3, HL-60, NB4), but not in those with low steadystate levels of MAPK activity (U937, KG1) (Figure 5b and data not shown). A net increase of more than $10 \%$ in the proportion of apoptotic cells was also observed in seven of nine primary AML samples (median, 17\%; range 11-72\%) after exposure to $10 \mu \mathrm{M}$ PD184352 (data not shown). In contrast, no reduction in cell viability or increase in apoptosis was observed in normal $\mathrm{CD} 34^{+}$progenitor cells from healthy donors $(n=3)$ following exposure to $10 \mu \mathrm{M}$ PD184352 (data not shown).

Interestingly, a strong, statistically significant correlation between steady-state levels of active MAPK and sensitivity to the growth-inhibiting effect of 
PD184352 was observed in AML cell lines $\left(R^{2}=0.8933, P=0.015\right.$; Figure 5d). Likewise, MEK inhibition-sensitive primary AML samples $(n=4)$ showed high levels of constitutive MAPK phosphorylation, whereas MAPK phosphorylation was barely detectable in resistant samples $(n=3, P=0.01)$ and in normal CD $34^{+}$progenitors (Figure $5 \mathrm{e}$ ).

\section{Discussion}

Here we provide evidence that small-molecule inhibitors of the MEK/MAPK pathway profoundly affect the growth of AML cell lines and primary AML samples with constitutive MAPK activation, but have little effect on cells with low steady-state MAPK activity. Cell growth was inhibited as a result of cell cycle arrest and induction of apoptosis, and resulted in the abrogation of leukemic, but not normal, hematopoietic cell clonogenicity. In addition, MEK inhibition profoundly sensitized leukemic cells to the proapoptotic effects of retinoids and chemotherapy.

Consistent with the proposed role of the RAS/MAPK pathway in the regulation of $G_{1} / S$ transition (25), both MEK inhibitors caused cell cycle arrest in sensitive AML cell lines and primary samples. Constitutive activation of the MEK/MAPK pathway results in decreased expression of the cyclin-dependent kinase inhibitor $\mathrm{p} 27^{\mathrm{Kip} 1}$ in different cell types, and recent data show that MAPK regulates $\mathrm{P} 27^{\text {Kip } 1}$ turnover at a posttranslational level $(26,27)$. Our data demonstrate that, in AML cell lines with constitutive MAPK activation, p27 Kip1 turnover is similarly regulated through MAPK-dependent mechanisms. Likewise, the observed decrease in $\mathrm{p} 21^{\text {Wafl/CIP1 }}$ levels in response to MEK inhibition is consistent with the reported ability of MAPK to transcriptionally induce p $21^{\text {Waf } 1 / \text { CIP1 } 1}$ expression (19). While downregulation of $\mathrm{p} 21^{\text {Waf1/CIP1 }}$ argues against its involvement in the observed inhibition of cell cycle progression, decreased levels of expression may play a role in the MEK inhibition-induced sensitization to the proapoptotic effects of retinoids and chemotherapy $(28,29)$. The potential clinical relevance of these findings is highlighted by recent reports that both low levels of $\mathrm{p} 27^{\text {Kip } 1}$ and high levels of $\mathrm{p} 21^{\text {Waf1/CIP1 }}$ confer a poor clinical outcome to AML patients $(30,31)$.

The evidence presented here suggests that MEK blockade alters the apoptotic balance by reducing the expression of both upstream $\left(\mathrm{Mcl}-1, \mathrm{Bcl}-\mathrm{X}_{\mathrm{L}}\right)$ and downstream (survivin) antiapoptotic proteins in cells that rely heavily on MAPK activation for their growth and survival. Although the relative importance of each of these players in the MEK inhibition-mediated lowering of the apoptotic threshold deserves further investigation, changes in survivin expression show the best correlation with the functional response to disruption of the MEK/MAPK module. These findings are consistent with recent observations showing that the MEK/MAPK pathway protects against apoptosis at the level of cytosolic caspase activation, without interfering with the release of cytochrome $c$ from mitochondria, strongly suggesting the involvement of an IAP-like molecule (32). Despite the above-described molecular changes, MEK inhibitors, at doses close to the $\mathrm{IC}_{50}$ for MAPK enzymatic activity, have cytostatic rather than cytotoxic effects, and higher doses are required to trigger apoptosis. However, MEK blockade sets the stage for increased sensitivity to other apoptotic stimuli, such as retinoids and DNA-damaging agents. Although MAPK may regulate $\mathrm{Bcl}-2$ antiapoptotic activity at a posttranslational level (33), we show here that MEK inhibition does not affect its protein expression; therefore, in the absence of additional cellular stresses, above-threshold levels of Bcl-2 can maintain cell viability even in the presence of low levels of other antiapoptotic players (such as Mcl-1 and survivin). This might in turn explain the proapoptotic synergism observed for the combination of ATRA treatment and
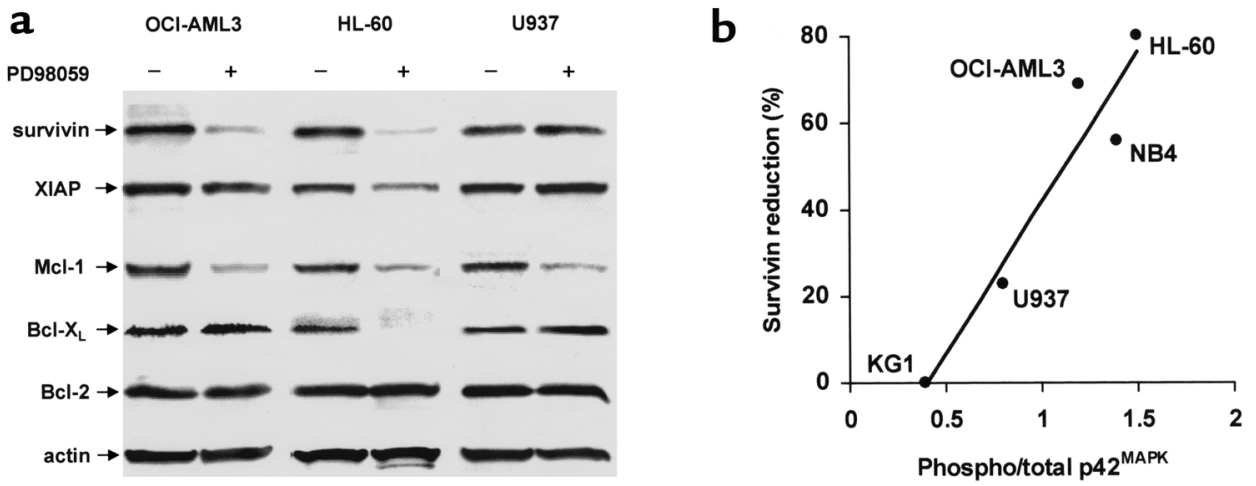

Figure 4

MEK inhibition downregulates antiapoptotic proteins of the IAP and Bcl-2 families. (a) OCI-AML3, HL-60, and U937 cells were cultured under standard conditions for 48 hours in the presence of DMSO or PD98059 (20 $\mu \mathrm{M})$, and then subjected to Western blot analysis with Ab's specific for the indicated antiapoptotic proteins of IAP (survivin, XIAP) and Bcl-2 (Mcl-1, Bcl-X $\mathrm{L}, \mathrm{Bcl}-2$ ) families. Results are representative of one of three independent experiments. (b) The MAPK phosphorylation status of AML cell lines was assessed by Western blot analysis. The ratio of phosphorylated to total $\mathrm{p} 42^{\mathrm{MAPK}}$ (Phospho/total $\mathrm{p} 42^{\mathrm{MAPK}}$ ) was then plotted against the percent reduction in survivin expression observed in the corresponding cell lines after PD98059 treatment, and regression analysis was performed $\left(R^{2}=0.9104, P=0.012\right)$. Results are representative of one of three independent experiments. 

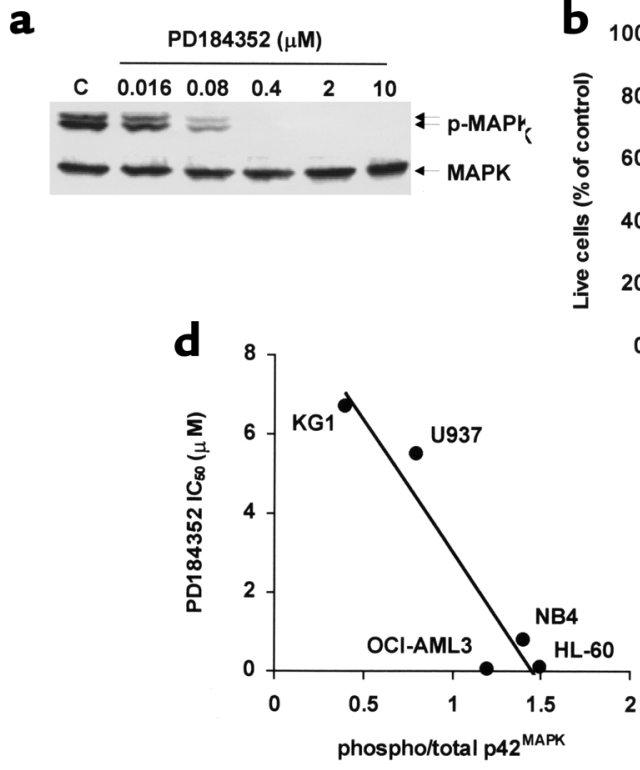
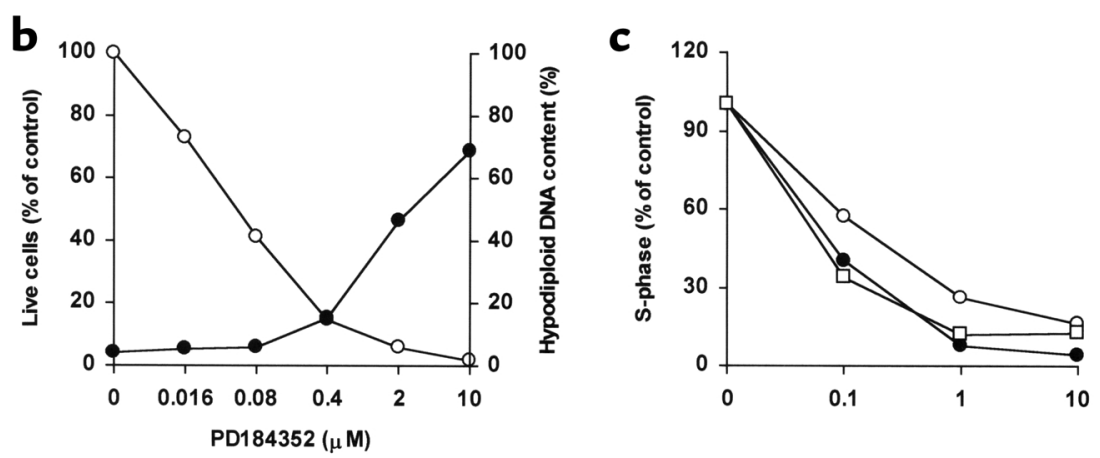

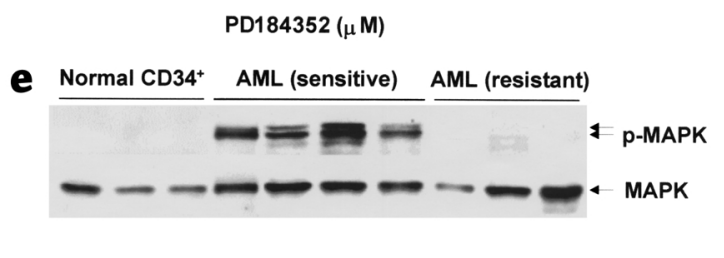

\section{Figure 5}

Effect of PD184352 on AML cell lines and primary samples. (a) OCI-AML3 cells were exposed to DMSO (C) or PD184352 for 6 hours and analyzed for MAPK phosphorylation. (b) OCl-AML3 cells were cultured in the presence of DMSO or PD184352. After 96 hours, cells were assessed for viability (open circles) and DNA content (filled circles). Results show viable cells in the PD98059-treated group, expressed as percentage of the viable cells in the DMSO-treated group, and percentage of cells with a hypodiploid DNA content, respectively, and are representative of one of three independent experiments. (c) OCI-AML3 (open circles), HL-60 (filled circles), and leukemic blasts from patient 19 (open squares) were cultured in the presence of DMSO or PD184352 for 48 hours and stained for DNA content. Results are expressed as percentage of S-phase cells in the DMSO-treated group. Cell line results are representative of one of three independent experiments. (d) The MAPK phosphorylation status of AML cell lines was assessed by Western blot. The ratio of phosphorylated to total $p 42^{\text {MAPK }}$ was then plotted against the IC $\mathrm{C}_{50}$ of PD184352 for the corresponding cell lines, and regression analysis was performed $\left(R^{2}=0.8933, P=0.015\right) .(\mathbf{e})$ $\mathrm{CD} 34^{+}$cells from healthy donors $(n=3)$ and blasts from AML patients $(n=7)$ were analyzed for phosphorylated MAPK by Western blot. Patient samples showing a greater than $25 \%$ decrease (from patients $14,19,21$, and 24 ) and a less than $25 \%$ decrease (from patients 17 , 20 , and 22) in cell viability after in vitro exposure to MEK inhibitors are labeled as sensitive and resistant, respectively.

MEK inhibition. In fact, ATRA efficiently decreases Bcl-2 expression (34, 35), whereas MEK inhibition downregulates downstream caspase inhibitors such as survivin, resulting in the simultaneous inhibition of complementary survival pathways, with synergistic effects on cell viability. This hypothesis is supported by our recent observation that simultaneous targeting of the MAPK and $\mathrm{Bcl}-2$ pathways results in the synergistic induction of apoptosis in AML cells (M. Milella, unpublished observations).

Based on its ability to lower the apoptotic threshold, disruption of the MEK/MAPK module has been proposed as a novel approach to the chemosensitization of cancer cells (36). Although our findings support this concept, they differ from previously reported results in two clinically relevant aspects. First, we found that only cell lines with constitutive activation of the MAPK pathway were sensitized to Ara-C-induced apoptosis, suggesting that the observed effect may depend on intrinsic rather than Ara-C-stimulated MAPK activity, as previously suggested (37). Second, as recently reported for paclitaxel (38), the Ara-C-potentiating effect was strictly sequence dependent. Ara-C followed by PD98059 substantially potentiated Ara-C-induced apoptosis, completely abrogating the ability of leukemic cells to recover from the cytotoxic insult, whereas the reverse sequence had a slight protective effect. The reduced efficacy of the combination of PD98059 followed by Ara-C may be related to the inhibition of cell cycle progression, which would result in decreased incorporation of Ara-C, and has potential implications for the design of clinical trials using MEKtargeted therapies as chemosensitizers.

The usefulness of mechanism-targeted therapeutic strategies, such as MEK inhibition, will ultimately depend on the ability to prospectively select those patients most likely to benefit from a specific intervention. Recent studies have shown that constitutive deregulation of specific pathways is the mechanism responsible for the sensitivity of cancer cells to signal-transduction inhibitors (39-41). In agreement with recent findings in different tumor models (17, 42), we observed a significant correlation between constitutive MAPK phosphorylation and sensitivity to MEK blockade-induced growth inhibition in both AML cell lines and primary AML samples. Along these lines, the lack of constitutive MAPK activation in normal hematopoietic progenitors (ref. 10 and Figure 5e) may explain their relative resistance to MEK inhibition, as observed here and in other recently published studies $(43,44)$, providing the basis for a selective antileukemic effect. 
Our overall results strongly support the hypothesis that disruption of the MEK/MAPK module, alone or in combination with retinoids and/or chemotherapy, may represent an effective and relatively specific therapeutic strategy for AML. The availability of orally active MEK inhibitors that have little or no systemic toxicity, such as PD184352, will allow further exploration of this approach in animal models and in human clinical trials.

\section{Acknowledgments}

This work was supported in part by grants from NIH (PO1 CA-55164, PO1 CA-49639, and CA-16672) and the Stringer Professorship for Cancer Treatment and Research (to M. Andreeff), and by an American Cancer Society International Fellowship for Beginning Investigators (to M. Milella). The authors wish to thank Judith S. Sebolt-Leopold (Department of Cell Biology, ParkeDavis Pharmaceutical Research) for kindly providing the MEK inhibitor PD184352, and Teresa McQueen for the separation of normal $\mathrm{CD} 34^{+}$hematopoietic progenitors.

1. Lowenberg, B., Downing, J.R., and Burnett, A. 1999. Acute myeloid leukemia. N. Engl.J Med. 341:1051-1062.

2. Look, A.T. 1997. Oncogenic transcription factors in the human acute leukemias. Science. 278:1059-1064.

3. Castilla, L.H., et al. 1999. The fusion gene Cbfb-MYH11 blocks myeloid differentiation and predisposes mice to acute myelomonocytic leukaemia. Nat. Genet. 23:144-146.

4. Rhoades, K.L., et al. 2000. Analysis of the role of AML1-ETO in leukemogenesis, using an inducible transgenic mouse model. Blood. 96:2108-2115. 5. Dash, A., and Gilliland, D.G. 2001. Molecular genetics of acute myeloid leukaemia. Baillieres Best Pract. Res. Clin. Haematol. 14:49-64.

6. Lewis, T.S., Shapiro, P.S., and Ahn, N.G. 1998. Signal transduction through MAP kinase cascades. Adv. Cancer Res. 74:49-139.

7. Hunter, T. 1997. Oncoprotein networks. Cell. 88:333-346.

8. Kim, S.C., et al. 1999. Constitutive activation of extracellular signal-regulated kinase in human acute leukemias: combined role of activation of MEK, hyperexpression of extracellular signal-regulated kinase, and downregulation of a phosphatase, PAC1. Blood. 93:3893-3899.

9. Towatari, M., et al. 1997. Constitutive activation of mitogen-activated protein kinase pathway in acute leukemia cells. Lenkemia. 11:479-484.

10. Hayakawa, F., et al. 2000. Tandem-duplicated Flt 3 constitutively activates STAT5 and MAP kinase and introduces autonomous cell growth in IL-3-dependent cell lines. Oncogene. 19:624-631.

11. Blalock, W.L., et al. 2000. A conditionally-active form of MEK1 results in autocrine transformation of human and mouse hematopoietic cells. Oncogene. 19:526-536.

12. Blalock, W.L., et al. 2000. Combined effects of aberrant MEK1 activity and BCL2 overexpression on relieving the cytokine dependency of human and murine hematopoietic cells. Leukemia 14:1080-1096.

13. Liu, Q., et al. 2000. Signal transduction and transforming properties of the TEL-TRKC fusions associated with $\mathrm{t}(12 ; 15)(\mathrm{p} 13 ; \mathrm{q} 25)$ in congenital fibrosarcoma and acute myelogenous leukemia. EMBO J. 19:1827-1838.

14. Carter, B.Z., Milella, M., Altieri, D.C., and Andreeff, M. 2001. Cytokine-regulated expression of survivin in myeloid leukemia. Blood. 97:2784-2790.

15. Dudley, D.T., Pang, L., Decker, S.J., Bridges, A.J., and Saltiel, A.R. 1995. A synthetic inhibitor of the mitogen-activated protein kinase cascade. Proc. Natl. Acad. Sci. USA. 92:7686-7689.

16. Alessi, D.R., Cuenda, A., Cohen, P., Dudley, D.T., and Saltiel, A.R. 1995. PD 098059 is a specific inhibitor of the activation of mitogen-activated protein kinase kinase in vitro and in vivo. J. Biol. Chem. 270:27489-27494.

17. Sebolt-Leopold, et al. 1999. Blockade of the MAP kinase pathway suppresses growth of colon tumors in vivo. Nat. Med. 5:810-816.

18. Estrov, Z., et al. 1995. Effect of interleukin-1 beta converting enzyme inhibitor on acute myelogenous leukemia progenitor proliferation. Blood. 86:4594-4602

19. Liu, Y., Martindale, J.L., Gorospe, M., and Holbrook, N.J. 1996. Regulation of $\mathrm{p} 21 \mathrm{WAF} 1 / \mathrm{CIP} 1$ expression through mitogen-activated protein kinase signaling pathway. Cancer Res. 56:31-35.

20. Slingerland, J., and Pagano, M. 2000. Regulation of the cdk inhibitor p27 and its deregulation in cancer. J. Cell Physiol. 183:10-17.

21. Xia, Z., Dickens, M., Raingeaud, J., Davis, R.J., and Greenberg, M.E. 1995.
Opposing effects of ERK and JNK-p38 MAP kinases on apoptosis. Science. 270:1326-1331.

22. Jarpe, M.B., et al. 1998. Anti-apoptotic versus pro-apoptotic signal transduction: checkpoints and stop signs along the road to death. Oncogene. 17:1475-1482.

23. Yen, A., Roberson, M.S., Varvayanis, S., and Lee, A.T. 1998. Retinoic acid induced mitogen-activated protein (MAP)/extracellular signal-regulated kinase (ERK) kinase-dependent MAP kinase activation needed to elicit HL-60 cell differentiation and growth arrest. Cancer Res. 58:3163-3172.

24. Davies, S.P., Reddy, H., Caivano, M., and Cohen, P. 2000. Specificity and mechanism of action of some commonly used protein kinase inhibitors. Biochem. J. 351:95-105.

25. Malumbres, M., and Pellicer, A. 1998. RAS pathways to cell cycle control and cell transformation. Front. Biosci. 3:d887-d912.

26. Lenferink, A.E., et al. 2000. Blockade of the epidermal growth factor receptor tyrosine kinase suppresses tumorigenesis in MMTV/Neu + MMTV/TGF-alpha bigenic mice. Proc. Natl. Acad. Sci.USA. 97:9609-9614.

27. Yang, H.Y., Zhou, B.P., Hung, M.C., and Lee, M.H. 2000. Oncogenic signals of HER-2/neu in regulating the stability of the cyclin-dependent kinase inhibitor p27. J. Biol. Chem. 275:24735-24739.

28. Wang, Z., et al. 1999. Dysregulation of the cyclin-dependent kinase inhibitor p21WAF1/CIP1/MDA6 increases the susceptibility of human leukemia cells (U937) to 1-beta-D-arabinofuranosylcytosine-mediated mitochondrial dysfunction and apoptosis. Cancer Res. 59:1259-1267.

29. Rosato, R.R., Wang, Z., Gopalkrishnan, R.V., Fisher, P.B., and Grant, S. 2001. Evidence of a functional role for the cyclin-dependent kinase-inhibitor $\mathrm{p} 21 \mathrm{WAF} 1 / \mathrm{CIP} 1 / \mathrm{MDA} 6$ in promoting differentiation and preventing mitochondrial dysfunction and apoptosis induced by sodium butyrate in human myelomonocytic leukemia cells (U937). Int. J. Oncol. 19:181-191.

30. Yokozawa, T., et al. 2000. Prognostic significance of the cell cycle inhibitor p27Kip1 in acute myeloid leukemia. Leukemia. 14:28-33.

31. Zhang, W., et al. 1995. High levels of constitutive WAF1/Cip1 protein are associated with chemoresistance in acute myelogenous leukemia. Clin. Cancer Res. 1:1051-1057.

32. Erhardt, P., Schremser, E.J., and Cooper, G.M. 1999. B-Raf inhibits programmed cell death downstream of cytochrome c release from mitochondria by activating the MEK/Erk pathway. Mol. Cell. Biol. 19:5308-5315.

33. Deng, X., Ruvolo, P., Carr, B., and May, W.S., Jr. 2000. Survival function of ERK1/2 as IL-3-activated, staurosporine-resistant Bcl2 kinases. Proc. Natl. Acad. Sci. USA. 97:1578-1583.

34. Andreeff, M., et al. 1999. Expression of bcl-2-related genes in normal and AML progenitors: changes induced by chemotherapy and retinoic acid. Lenkemia. 13:1881-1892.

35. Ketley, N.J., Allen, P.D., Kelsey, S.M., and Newland, A.C. 1997. Modulation of idarubicin-induced apoptosis in human acute myeloid leukemia blasts by all-trans retinoic acid, $1,25(\mathrm{OH}) 2$ vitamin $\mathrm{D} 3$, and granulocytemacrophage colony-stimulating factor. Blood. 90:4578-4587.

36. Dent, P., and Grant, S. 2001. Pharmacologic interruption of the mitogen-activated extracellular- regulated kinase/mitogen-activated protein kinase signal transduction pathway: potential role in promoting cytotoxic drug action. Clin. Cancer Res. 7:775-783.

37. Jarvis, W.D., et al. 1998. Evidence for involvement of mitogen-activated protein kinase, rather than stress-activated protein kinase, in potentiation of 1-beta-D- arabinofuranosylcytosine-induced apoptosis by interruption of protein kinase C signaling. Mol. Pharmacol. 54:844-856.

38. Yu, C., Wang, S., Dent, P., and Grant, S. 2001. Sequence-dependent potentiation of paclitaxel-mediated apoptosis in human leukemia cells by inhibitors of the mitogen-activated protein kinase kinase/mitogenactivated protein kinase pathway. Mol. Pharmacol. 60:143-154.

39. Shayesteh, L., et al. 1999. PIK3CA is implicated as an oncogene in ovarian cancer. Nat. Genet. 21:99-102.

40. Druker, B.J., et al. 1996. Effects of a selective inhibitor of the Abl tyrosine kinase on the growth of Bcr-Abl positive cells. Nat. Med. 2:561-566.

41. Brognard, J., Clark, A.S., Ni, Y., and Dennis, P.A. 2001. Akt/protein kinase $\mathrm{B}$ is constitutively active in non-small cell lung cancer cells and promotes cellular survival and resistance to chemotherapy and radiation. Cancer Res. 61:3986-3997.

42. Hoshino, R., Tanimura, S., Watanabe, K., Kataoka, T., and Kohno, M. 2000. Blockade of the extracellular signal-regulated kinase pathway induces marked G1 cell cycle arrest and apoptosis in tumor cells in which the pathway is constitutively activated: Up-regulation of p27Kip1. J. Biol. Chem. 276:2686-2692.

43. Fichelson, S., et al. 1999. Megakaryocyte growth and development factor-induced proliferation and differentiation are regulated by the mitogen-activated protein kinase pathway in primitive cord blood hematopoietic progenitors. Blood. 94:1601-1613.

44. Morgan, M.A., Dolp, O., and Reuter, C.W. 2001. Cell-cycle-dependent activation of mitogen-activated protein kinase kinase (MEK-1/2) in myeloid leukemia cell lines and induction of growth inhibition and apoptosis by inhibitors of RAS signaling. Blood. 97:1823-1834. 